\title{
Intelligent window and its application to anti haze
}

\author{
Yumeng Wang ${ }^{1, a}$, Ruonan $\mathrm{Xue}^{2, \mathrm{~b}}$ and $\mathrm{Xue} \mathrm{We}^{2, \mathrm{c}}$ \\ ${ }^{1}$ School of North China Electric Power University(Baoding), Hebei 071000, China; \\ ${ }^{2}$ School of North China Electric Power University(Baoding), Hebei 071000, China. \\ aydfz2010@qq.com, b467054132@qq.com, c1574222456@qq.com
}

Keywords: WIFl; anti-fog and haze; automatic window opener; intelligent

\begin{abstract}
The current urban air quality is deteriorating. Especially in northern China, air quality $\mathrm{h}$ as become a hot topic. How to improve indoor air quality raises our interests. Our research team has developed the device switches the anti-haze window automatically under WIFI control by using filtering screens, air quality monitor, automatic window opener, remote control devices such as mobile phones.
\end{abstract}

\section{Introduction}

Currently, the air pollution in some cities in northern China is serious and people pay more and more attention to air quality. The number of people who died of asthma caused by indoor air pollution is over 100 thousand each year, 35 percent of whom

are children. The World Health Organization has listed the indoor environmental pollution as o ne of the top ten killers of human health, so we have to pay full attention to our indoor environme ntal quality.

There are currently two major ways to control air pollution: First, reducing pollution sources, and second,cleaning or purifying air. If the outdoor environment is good enough, it is possible to improv e the content of oxygen in the air and reduce the concentration of toxic gases by exchanging air bet ween the outside and inside of the room. However, in areas which are contaminated severely, the air outdoors is much more polluted than that indoors, it will do harm to people's health if they open the windows directly. The air purifiers in the market now are expensive and high energy consumption. Since the windows and doors need to be closed when using, the oxygen content will reduce and the carbon dioxide content will increase. Therefore, we want to design a set of equipment which aims at purifying the air indoors by opening the windows automatically under the control of the smart phone with WIFI connection.

\section{Design ideas}

Air quality monitor detects the air indoors and uploads the test result to the smart phone. If the result shows that the PM2.5 concentration is higher than $115 \mathrm{ug} / \mathrm{m}^{3}$, then the window can be opened automatically under the control of a smart phone. The information detected by the air quality monitor can be delivered to specific application installed in the smart phone with the help of WIFI connection. The air outdoors will be filtered by filtering screens anchored beside the window before it enters into the insides. After a while, if the test result shows that the PM2.5 concentration is lower than $115 \mathrm{ug} / \mathrm{m}^{3}$, the window can be closed intelligently. 
Table 1

\begin{tabular}{|c|c|c|c|}
\hline \multicolumn{4}{|c|}{ Air quality standards $\left(1 \mathrm{mg} / \mathrm{m}^{3}=1000 \mathrm{ug} / \mathrm{m}^{3}\right)$} \\
\hline $\begin{array}{l}\text { PM2.5concentration(unit: } \\
\mathrm{ug} / \mathrm{m}^{3}\end{array}$ & Air pollution index & Pollution levels & Health advice \\
\hline $0-35$ & $0-50$ & excellent, & $\begin{array}{l}\text { All types of people can do normal } \\
\text { activities }\end{array}$ \\
\hline $36-75$ & $50-100$ & good: & $\begin{array}{l}\text { Very few unusually sensitive } \\
\text { people should reduce outdoor } \\
\text { activities: }\end{array}$ \\
\hline $76-115$ & $150-200$ & Light pollution & $\begin{array}{l}\text { Children, the elderly and patients } \\
\text { with heart or respiratory disease } \\
\text { should reduce prolonged or } \\
\text { high-intensity outdoor exercises. }\end{array}$ \\
\hline $116-150$ & $200-250$ & Moderate pollution & $\begin{array}{l}\text { Children, the elderly and patients } \\
\text { with heart or respiratory diseases } \\
\text { should reduce prolonged or } \\
\text { high-intensity outdoor exercises; } \\
\text { general population should reduce } \\
\text { the amount of outdoor activities } \\
\text { moderately. }\end{array}$ \\
\hline $151-250$ & $250-300$ & Severe pollution & $\begin{array}{l}\text { Children, the elderly and patients } \\
\text { with heart or respiratory diseases } \\
\text { should stay indoors, stop outdoor } \\
\text { sports; general population should } \\
\text { reduce the amount of outdoor } \\
\text { activities. }\end{array}$ \\
\hline More than 250 & More than 300 & Serious pollution & $\begin{array}{l}\text { Children, the elderly and patients } \\
\text { with heart or respiratory diseases } \\
\text { should stay indoors and avoid } \\
\text { physical consumption; general } \\
\text { population should avoid doing } \\
\text { outdoor activities. }\end{array}$ \\
\hline
\end{tabular}

(1).Filter screens

This device is made of Polypropylene Melt-blown filter sieve which is charged with static electricity for catching dust. By using high flow PP as basic material and adding special auxiliary, the filter screen can be produced by melting Co-extrusion granulation. The device has a high charge entrapment both in depth and density and is able to improve filtration efficiency and thermal fade resistance performance. A thermal aging test shows that the filtration loss is less than 0.02 percent (70 degrees to store 72 hours). Laboratory test shows that the purification efficiency of the dust whose diameter is smaller than $2.5 \mathrm{MM}$ is higher than 95 percent and the device maintains excellent low resistance. Therefore, people can be away from the pollution outdoors by using this kind of filter screen made of special material. 


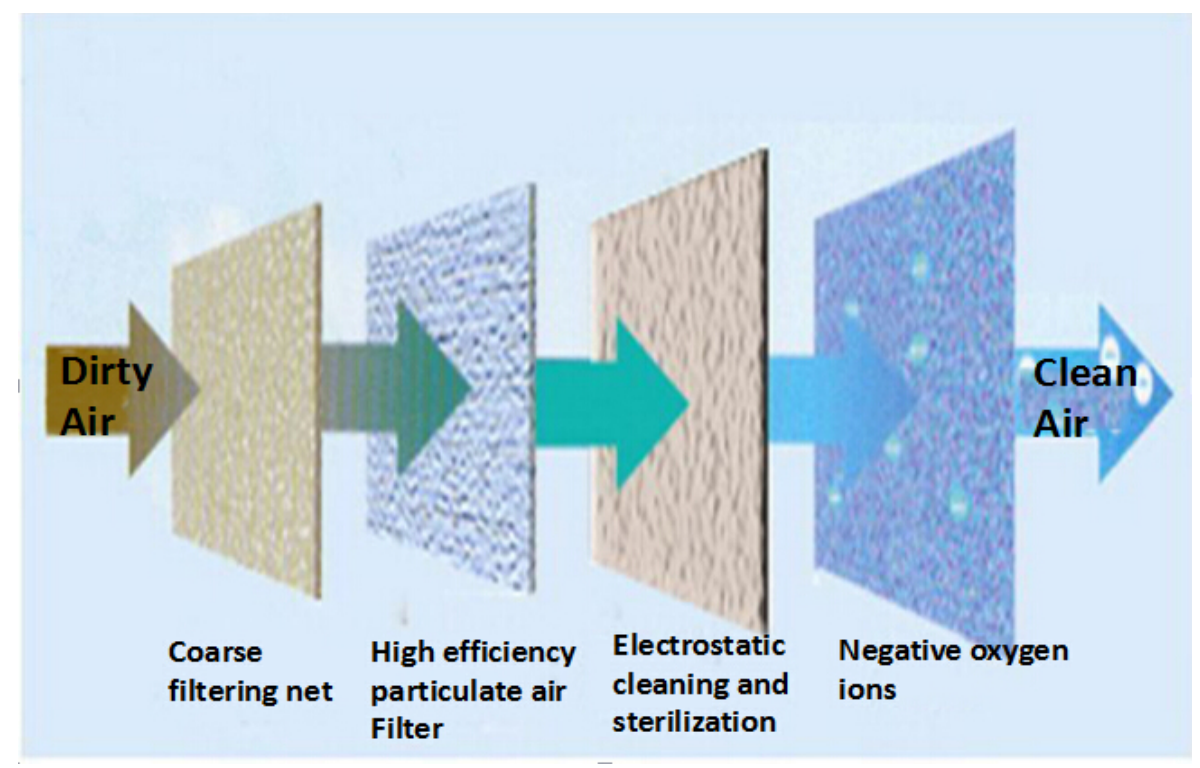

(2). Air quality monitor

Fig 2

The stability of the device can be guaranteed by using laser optical standards rubato.The device accomplishes the compatibility of particle cutters of PM10,PM5, PM2.5,TSP. By using filter sampler, dust monitor is compatible with filter sampling.The information of the components of thick and thin particles in air can be analyzed and the airborne dust concentration conversion factor (K-Factor) can be calculated. The constant current controller is designed to keep flow amounts constant and the correctness of cutting diagram. The special designed air curtain prevents the pollution of the core components -optical system.

The instrumental measuring accuracy can be improved by automatic Zero Adjustment realized by computer software.

(3). Design and development of window opening device

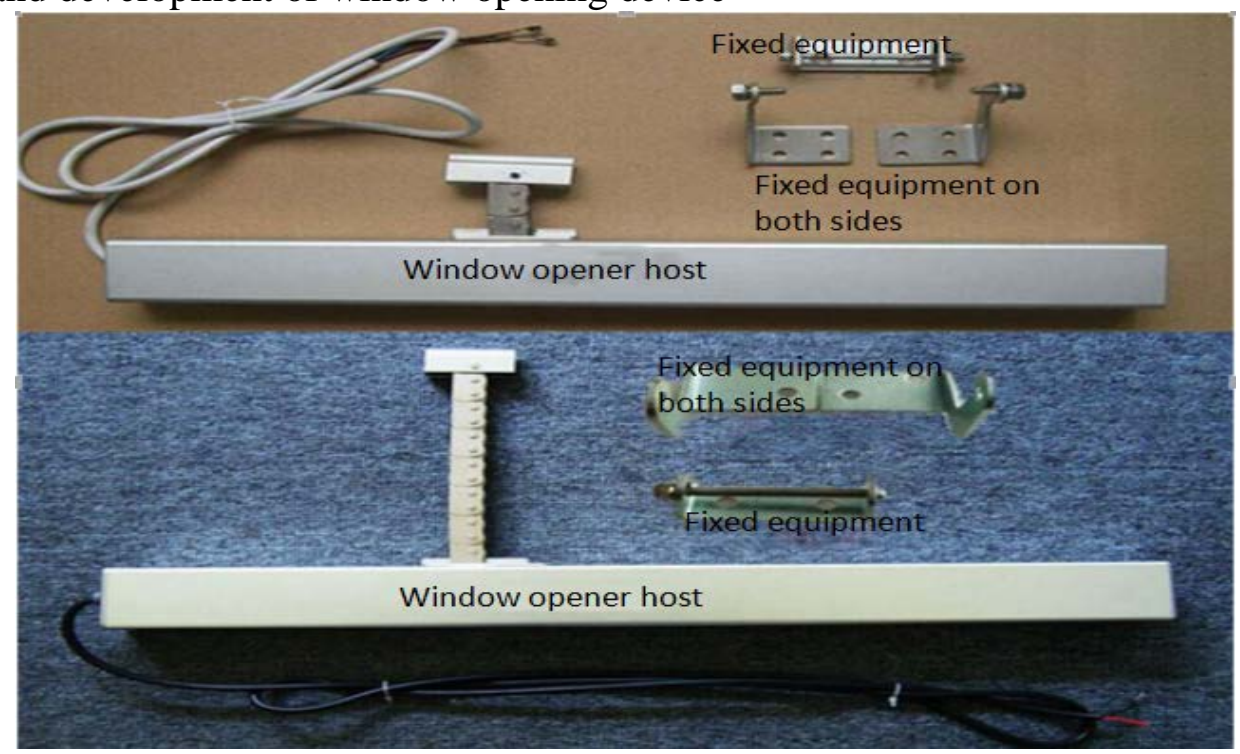

(4).WiFi control module

Fig 3

The transceiving module is EMW3160 development Kit which is made by MXCHIP. This kit includes EMB-380-S2 evaluation boards and Internet of things WiFi module. Windows can be controlled by the application installed in the smart phone with WIFI connection. 


\section{Prospect}

According to the survey, the number of residents in Chinese large and medium urban areas has reached 300 million now. We assume that every 5 people make up one family unit and demand one air purifier product, so that there will be a potential market of about 60 million products.

Nowadays, the current indoor air purification product market has a certain scale and is into the growth period. The endless stream of products like air fresheners, ultrasonic humidifiers, photo-catalyst air purifier, negative oxygen ion air purification products has made indoor environmental purification treatment a major new industry in China. Some department forecasts that based on the current industry scale of Purification and Treatment of Indoor Environment in China of more than 20000 million yuan, a compound annual growth rate will be 28 percent in the next few years.

\section{Innovating points}

(1) Intelligent :It will be more direct and accurate to monitor the air quality, harmful gas and the concentration of PM2.5. In addition, with the rapid development of the intelligent mobile terminal, the user has easier access to the surrounding air quality. The smart phone can collect the data of real-time environmental temperature, humidity and PM2.5 values from the built-in air sensor using WIFI data transmission module. The data can also be transmitted to cloud network for future research and analysis. More importantly, the user can automatically control the window ease of use and it helps reduce manpower greatly.

(2) Health and environmental protection :Before the outdoor air is transmitted to the room, the filter screen will filter the dust and other air pollutants. Thus, it is possible to eliminate various kinds of diseases caused by a closed environment.

(3)Energy saving :Filter screens are cost-effective. There is no need of charging and it will not make any noise. The air purification effect can last for a long time. However, the air purifiers in current market are expensive and have several disadvantages in the process of using such as high energy consumption, noise, radiation. It can only be effective when the windows are closed and the concentration of oxygen will decline after using them for a long time.

\section{Summary}

With the help of the WIFI connection and the application installed in the smart phone, it is easy to control the window automatically according to the Real-time Supervisory Data. The air outside can be filtered using the filter screen, so that it is economical and convenient to keep the air fresh and clean enough in areas where there are a lot of people and the air needs to be flowed frequently. If the whole equipment is widely put into practical use, it will create economical, social and environmental effects.

\section{References}

[1] Li Ling-juan,Ding Liang.Research on Multi-Hop Routing Algorithm of Wireless Sensor Network[J] COMPUTER TECHNOLOGY AND DEVELOPMENTe.2010, 20(6): 55-58.

[2] Hou Rong-hui,Shi Hao-shan,Yang Shao-jun.New Data-Centric Qos Routing Protocol for Wireless Sensor Netwoks [J].CHINESE JOURNAL OF SENSORS AND ACTUATORS, 2006, 19(6): 2732-2737.

[3] Xie Xi-ren ,The computer network[M],Fifth edition. Electronic Industry Press .2008

[4]Wu Gong-yi,Wu Ying.Introduction to the Internet of things.Beijing: higher education press.2012, 7

[5]Peng $\mathrm{Li}$, Based on the case of an introduction to the Internet of things , Beijing: Chemical Industry Press.2012,10. 